\title{
HSDPA Performance in a Mixed Traffic Network
}

\author{
Martin Wrulich*, Werner Weiler ${ }^{\S}$ and Markus Rupp* \\ *Institute of Communications and Radio-Frequency Engineering, Vienna University of Technology \\ Email: \{mwrulich,mrupp\}@nt.tuwien.ac.at, Web: http://www.nt.tuwien.ac.at/research/mobile-communications/ \\ $\S$ mobilkom austria AG, Email: w.weiler@mobilkom.at, Web: http://www.mobilkom.at
}

\begin{abstract}
One of the advantages in an existing WCDMA network is that HSDPA (High Speed Downlink Packet Access) can be operated within an existing Release 4 (Release 99) $5 \mathrm{MHz}$ band, thus allowing for sharing the power amplifier and spreading codes at the Node-B between the HS-DSCH (High Speed Downlink Shared Channel) and the Release 4 downlink channels (DCHs). In this paper, we investigate the throughput performance of HSDPA in a network, in which also Release 4 traffic is present by utilizing a system level modeling of the HSDPA transmission if a Rake receiver is used, together with a coarse modeling of the Release 4 performance. By numerical simulations, we are able to deduce the optimum power split between HSDPA and Release 4 to maximize the overall cell throughput. The obtained results offer a more sophisticated understanding of the HSDPA network performance, and can be used by network operators for cell operation planning.
\end{abstract}

\section{INTRODUCTION}

High speed downlink packet access (HSDPA) has been introduced in Release 5 of 3GPP UTRAN [1]. The key idea of the HSDPA concept is to increase the packet data throughput compared to the rates provided by the Release 4 (also often called Release '99) WCDMA specifications. The fundamental techniques used are link adaptation and fast physical (L1) retransmission combining. In particular, adaptive modulation and coding (AMC), extensive multicode operation and a fast and spectrally efficient retransmission strategy replace the two most fundamental features of WCDMA, variable spreading factors and fast power control. Furthermore, a shorter transmission time interval (TTI) of $2 \mathrm{~ms}$ can be used. The shared transport channel used for the HSDPA operation is the high-speed downlink shared channel (HS-DSCH) which is multiplexed to the high-speed physical downlink channels (HS-PDSCHs), each with its own spreading code.

HSDPA is designed so that it can be operated within an existing $5 \mathrm{MHz}$ band of Release 4 , already utilized by dedicated channels $(\mathrm{DCH})$, thus allowing for sharing the power amplifier at the Node-B. This implies that one part of the available Node-B power and spreading codes is used for the DCHs and another part can be used for the HSDPA operation. This specific mode of operation is of particular interest for the network operators in the phase of the HSDPA deployment, and even if HSDPA is widely installed, a mixed carrier operation is more cost-efficient for cells that are not fully loaded.

Despite the significance of the study of the network performance behavior in such a scenario, not much work has been performed in order to assess the average user throughput (or equivalently the total cell throughput), or to derive specific recommendations for the network planning process. The available work discusses only a very limited part of the whole topic, see e.g. [2], where furthermore only the Veh-A ITU channel profile has been considered, which is a fairly unusual for most of the HSDPA traffic occurring in a network.

In our paper, we investigate the average user throughput in a mixed carrier scenario (Release 4 DCH and HSDPA) and deduce the optimum Node-B power split under different conditions by means of snapshot based network simulations. The results can be used by network operators for the cell operation planning, e.g. to decide whether the deployment of a power amplifier with higher capacity, or the introduction of a second frequency solely for the HSDPA traffic is more feasible, depending on the actual Release 4 load of the cell.

The paper is organised as follows: in Section II our systemlevel model for the investigation of the average HSDPA user throughput is explained. Section III outlines some specific details of our simulator, after which Section IV presents and discusses the numerical results obtained. Section V concludes the paper.

\section{SySTEM MODEL}

\section{A. Channel modeling}

The radio propagation model we use throughout this paper considers basically three different parts: deterministic pathloss $d$, shadow fading $s$, and small-scale fading with multiple paths and no correlation in time, since our simulations are snapshot based with no correlations in between. Accordingly, the channel coefficient between the base station and the user equipment can be written as

$$
h(\tau)=d \cdot s \cdot \sum_{l=1}^{L} \sqrt{p_{l}} \cdot f_{l} \cdot \delta\left(\tau-\tau_{l}\right),
$$

where the random variables $f_{l}$ represent $L$ independent Rayleigh fading processes at fixed time slots, $\delta$ denotes the Dirac function and $p_{l}, \tau_{l}$ are the relative power and delay of the multipath components, according to the ITU-R channel profiles [3].

The deterministic path-loss $d$ depends only on the distance $r$ between the base station and the user equipment, which we model according to the COST231 Model [4]. The shadow fading $s$ is modelled by a lognormal random variable with zero mean and $\sigma_{s}=8 \mathrm{~dB}$, with no correlation in time.

\section{B. HSDPA modeling}

To assess the performance of HSDPA on network level, the structure of the network, the user distribution, and many 
other influencing factors have to be simulated. Unfortunately, an exact evaluation of the HSDPA network performance by means of individual link level simulations (for each user and base-station) in this context would result in an unfeasible computational complexity. Thus, to avoid this computational demand but nevertheless represent the HSDPA performance in an accurate way, we modelled the individual links through a simplified system level description.

Basically, the modeling splits up into two parts: one describing the channel quality as observed by the receiver (the so-called link-measurement model) and one describing the biterror/decoding performance (the so-called link-performance model), see e.g. [5]. For the link-measurement model, we assumed a standard single antenna Rake receiver at the user terminal, because in current networks, most of the terminals are equipped with such.

As channel quality metric we evaluated the signal-to-noiseand-interference ratio (SINR) after Rake-combining and despreading (i.e. on symbol level) for each user $u$ in the cell, according to

$$
\mathrm{SINR}_{u}=\sum_{i=1}^{N_{F}} \frac{\mathrm{SF} \cdot \frac{P_{\mathrm{HS}-\mathrm{DSCH}}}{\gamma} \cdot\left|h_{i}\right|^{2}}{P_{\text {intra, residual }}+P_{\text {inter }}+P_{\text {noise }}},
$$

where $\mathrm{SF}=16$ denotes the spreading factor, $P_{\mathrm{HS}-\mathrm{DSCH}}$ is the power used for transmitting the HS-DSCH, $P_{\text {intra, residual }}$ and $P_{\text {inter }}$ represent the residual intracell interference (in the downlink) and the transmitted interfering power from the neighbouring base stations, respectively, and $P_{\text {noise }}$ denotes the noise power as seen at the receiver. The residual intracell interference (after Rake processing), arriving at the receiver from the serving base station, is given by [6], $P_{\text {intra,residual }}=$ $P_{\text {intra }} \cdot \sum_{\substack{l=1 \\ l \neq i}}^{L}\left|h_{l}\right|^{2}$, where $L$ denotes the total number of taps for the current channel realisation, denoted by $h_{l}$, and $P_{\text {intra }}$ is the total power transmitted in the serving cell.

The structure of the Rake receiver is implicitly reflected in Equation (2), because the useful signal power in the numerator is added up, whereas in the denominator it is cancelled out from the interference power, which is consecutively done for all $N_{F}$ available fingers. We assume perfect channel state information (CSI) at the receiver so that the receiver weights and the location of the fingers can be chosen perfectly. Accordingly, only the squared absolute values of the channel coefficients (for each tap), $\left|h_{i}\right|^{2}$ occur in the equation. Also note that the HS-DSCH is a transport channel [7], and that in the physical transmission it is split into several highspeed physical downlink shared channels (HS-PDSCH), each spread with its own spreading sequence. Throughout this paper, we assume that the transmission power of the HSDSCH is divided equally between all used HS-PDSCH, i.e. $P_{\mathrm{HS}-\mathrm{PDSCH}, i} \equiv P_{\mathrm{HS}-\mathrm{PDSCH}}=P_{\mathrm{HS}-\mathrm{DSCH}} / \gamma$, with $\gamma=1, \ldots, 15$ representing the number of assigned spreading codes.

For the link-performance, we chose an analytical approach based on link-level simulations, as in [8]. In principle, linklevel simulations have been used to provide a limited number of points in an SINR versus BLER plot for a specific transport format. In our simulations, we consider the case that the desired user (for HSDPA) always gets the full available transmission power, and there is enough data to transmit (full buffer). Accordingly, we assume that the scheduler in the Node-B decides to serve the user with the transport format that is specified by the reported CQI (see [9]). Therefore, we only need a link performance modelling for the transport formats of each mobile category class, given by the possible CQI values. As e.g. in [10], it turns out that under AWGN conditions, the link-level results, utilizing a standard Rake receiver together with turbo coding, can analytically be well approximated by

$$
\mathrm{BLER}=\left\{10^{\left(2 \frac{\mathrm{SINR}-1.03 \mathrm{CQI}+5.26}{\sqrt{3}-\log _{10} \mathrm{CQI}}\right)}+1\right\}^{-\frac{1}{0.7}},
$$

where in contrast to [10], we use the post-despreading symbollevel SINR, as we defined in (2). The coding rate of the turbo-code has been varied from 0.21 to 0.75 in the link-level simulations to reflect typical coding rates for the different CQI values [11].

One may argue that AWGN link-level simulations do not apply for frequency selective fading network simulations, e.g. based on the ITU-R channel models [3]. In system level simulations of adaptive systems, like for HSDPA, however, each scheduling that is based on channel quality information effectively influences the channel statistics. For example, if a maximum throughput scheduler is implemented, each user will only be served, if he observes a "good" channel. Since system level decisions are not known a priori at link level, an adequate simulation is impossible and thus the receiver performance has to be appropriately approximated, which we did by the simplification of using the AWGN curves.

Furthermore, it has to be noted that due to the snapshot based simulation approach we chose, we did not model any HARQ retransmission gains. The BLER is considered fully in the evaluation of the throughput.

\section{Release 4 modeling}

In our investigations we were mainly interested in a prediction of the achievable HSDPA user data rates in dependence of a given Release 4 DCH load in the cell. Accordingly, we modelled the Release 4 traffic only coarse. The whole network traffic generated by the DCHs is just described by the already mentioned load of the cell, given by a transmission power value, $P_{\mathrm{DCH}}$. Of course, this prevents an exact modeling of the fast power control of the users - since $P_{\mathrm{DCH}}$ just describes the average transmission power - and the possibility of predicting the Release 4 cell throughput at the actual power level is very limited.

Nevertheless, we wanted to roughly estimate the total DCH cell throughput in order to be able to predict the overall cell throughput. An almost suitable concept for our needs is explained in [1], where the estimation of the average needed base station transmission power for a given DCH traffic, based on the downlink load factor is investigated. We adapted the idea therein to obtain a suitable description for our simulation environment, which we will sketch here. 
According to [12], the link quality equation for $\mathrm{DCH}$ downlink connections, in a cell $m$, is given by

$$
\varrho_{i}=\frac{W p_{i} \frac{1}{L_{m, i}}}{R_{i}\left[\left(1-\alpha_{i}\right) \cdot \frac{P_{\text {intra }}}{L_{m, i}}+P_{\text {intra }} \cdot \sum_{\substack{n=1 \\ n \neq m}}^{N} \frac{1}{L_{n, i}}+P_{N}\right]},
$$

where $\varrho_{i}$ denotes the required $E_{b} / N_{0}$ (bit level SINR) for a specific user $i$, requesting a service with bit rate $R_{i}$. The chip rate of the system is given by $W, L_{m, i}$ represents the path loss of base station $m$ to $\mathrm{DCH}$ user $i, \alpha_{i}$ stands for the orthogonality factor as observed by user $i, P_{\text {intra }}$ is again the total transmit power of the BS and $P_{N}$ stands for the received thermal noise power. The total number of considered base stations is given by $N$. We are mainly interested in the $p_{i}$, representing the required transmission power of base station $m$ in order to supply user $i$ with the defined service, because the sum of it equals the DCH cell load, i.e. $\sum_{i=1}^{I} p_{i}=P_{\mathrm{DCH}}$, with $I$ denoting the total number of active DCH users in the cell.

We assumed the same service for all DCH users in the cell, i.e. $\varrho_{i} \equiv \varrho$ and $R_{i} \equiv R$. In analogy to [1], we introduced the own to other cell interference ratio, given by

$$
\mathcal{O}=\sum_{\substack{n=1 \\ n \neq m}}^{N} \frac{L_{m, i}}{L_{n, i}}
$$

but, since we did not want to model the DCH users explicitly, we substituted $\mathcal{O}$ and $L_{m, i}$ by their average over the cell area, i.e. $\mathcal{O} \rightarrow \overline{\mathcal{O}}$ and $L_{m, i} \rightarrow \bar{L}_{m}$.

The orthogonality factor, $\alpha$, can be calculated in a simple way as described in [6],

$$
\alpha_{i}=1-\left(\sum_{l=1}^{L} \frac{\left|\beta_{l, i}\right|^{2}}{1-\left|\beta_{l, i}\right|^{2}}\right)^{-1},
$$

with $\beta_{l, i}=\sqrt{p_{l}} f_{l, i}$ describing the complex valued coefficient of the channel tap $l$, for each user $i$, respectively. The user dependency in this case is only introduced because of the small-scale fading channel statistics. In complete analogy to the simplifications already introduced above, we substitute the orthogonality factor $\alpha_{i}$ by its average $\alpha$, which is achieved by using the average channel tap values $\mathbb{E}\left\{\left|\beta_{l}\right|^{2}\right\}=p_{l}$, determined by the ITU-R channel profiles.

With this simplifications, after some algebra, the number of DCH users that can be served given a specific data rate, e.g. a $384 \mathrm{kbit} / \mathrm{s}$ connection, can be derived as

$$
I=\frac{P_{\mathrm{DCH}}}{P_{\text {intra }} \cdot \frac{\varrho R}{W} \cdot[(1-\bar{\alpha})+\mathcal{O}]+P_{N} \cdot \frac{\varrho R}{W} \cdot \bar{L}_{m}} .
$$

\section{Power split}

The total available transmit power in each cell is shared between HSDPA and DCH users. We expressed the total intracell transmission power as

$$
P_{\text {intra }}=P_{\mathrm{DCH}}+P_{\mathrm{HS}-\mathrm{DSCH}}+P_{\mathrm{other}},
$$

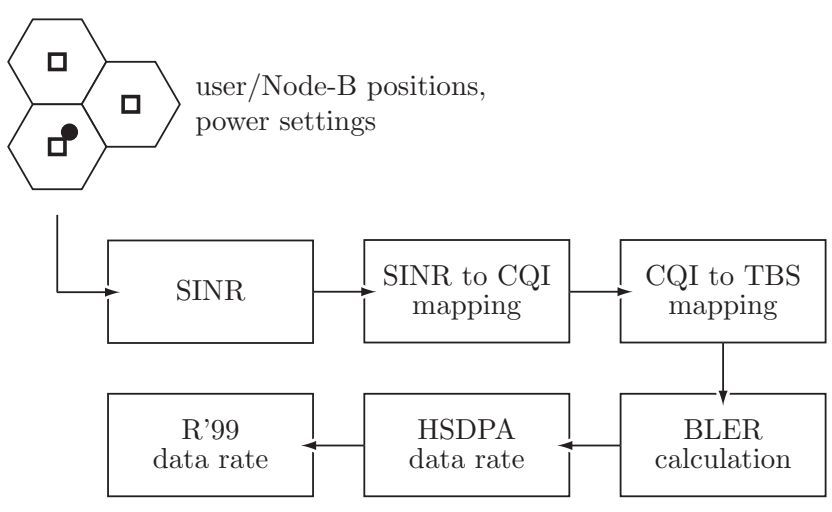

Fig. 1. Overview of the basic steps in the snapshot system level simulator.

where $P_{\text {other }}$ incorporates the power from other needed common channels, e.g. the common pilot channel (CPICH). Accordingly, both, Equation (2) and Equation (7) depend on the transmission power of the DCH and the HSDPA traffic.

In principle, there are two different possibilities of allocating the power of HSDPA in the base station downlink power budget [13]: (1) the RNC can dynamically allocate HSDPA power by sending Node-B application part (NBAP) messages to the base station, which effectively keeps the HSDPA power at a fixed level, whereas the DCH power varies according to the fast closed loop power control. The other option is (2) that no NBAP messages are sent and the base station is allowed to allocate all unused power for HSDPA, which better utilises the power amplifier.

\section{Simulation Details}

\section{A. Simulation Approach}

For our analysis, we are mainly interested in the evaluation of the average HSDPA user data rate, as well as the average HSDPA cell data rate. Therefore, we implemented a simplified simulation approach that is based on uncorrelated snapshot simulations of the current interference situation in the network. Of course, this approach prevents the evaluation of HARQ retransmissions and the analysis of scheduler algorithms.

Figure 1 shows a coarse overview of the snapshot system level simulator, we implemented in MATLAB. With a fixed network situation, the SINR can be calculated according to Equation (2), which then is mapped to the CQI value that will be reported with the UE that is currently under investigation. The mapping is given by $\mathrm{CQI}=\operatorname{SINR}[\mathrm{dB}]+3.5$, as defined in [11]. The evaluated CQI can then successively be mapped to a transport block size (TBS), which denotes the maximum amount of data that can be transmitted via the network in one TTI of $2 \mathrm{~ms}$ to the UE without exceeding a BLER of 0.1 in average (see [7]). Of course, the actual BLER value differs from the average, so that we calculate the HSDPA user data rate according to

$$
R_{u}=\mathrm{TBS} \cdot \frac{1}{2 \mathrm{~ms}} \cdot(1-\mathrm{BLER}),
$$

which is consecutively averaged over fading realizations. 
Since we assume that the desired user is served with the transport format according to the reported CQI value, we implicitly also assume that the user gets the full available HSDPA transmission power. This means that no other user is served in parallel, and hence we obtain the maximum user throughput. Thus, by averaging over the cell, we can evaluate the average user performance within the cell, which we will denote "HSDPA user data rate" in the following. Within the simulator, the intercell interference is modelled by assuming a homogeneous network operation, i.e. the actual transmission power of the neighbouring Node-Bs is chosen exactly equal to the power of the serving Node-B.

The Release 4 data rate is evaluated based on the modeling in Section II-C, where the service that is requested by all users is adjusted by the required data rate of it. This means that we can calculate the average cell data rate corresponding to the actual power settings of the network by consecutively multiplying the calculated number of served users by the specified data rate.

\section{B. Network Model}

Due to its general applicability, we decided in favor of a hexagonal network layout, organized in a serving cell and two rings of neighbouring base stations (19 cells in total), to be implemented in the simulator. This layout is described as cellular layout 2 in [14]. The antennas used at the Node-Bs that split the cell area into three sectors are modelled by

$$
L_{\text {ant }}^{i}\left(\theta^{i}\right)=\bar{L}_{\text {ant }}^{i} \cdot 10\left(-\frac{1}{10} \min \left\{12\left(\frac{\theta^{i}}{70^{\circ}}\right), 20\right\}\right),
$$

where we denoted the mean antenna gain by $\bar{L}_{\text {ant }}^{i}=14 \mathrm{dBi}$, and the index $i$ represents the sector.

\section{REsults}

We now present the simulation results of the modelled system, which can serve for identifying possible improvements of the performance by adjusting the power and code settings in the network. As already mentioned in Section II-D, there are two possibilities of controlling the power reserved for the HS-DSCH traffic, so we simulated both scenarios.

\section{A. RNC controlled HS-DSCH power}

First, we performed some simulations with the HS-DSCH power controlled by the RNC and with a fixed Release 4 load. The simulation parameters are given in Table I.

The results of a simulation carried out for a Release 4 load of $20 \%$ (of the maximum Node-B power, i.e. $P_{\mathrm{DCH}}=$ $P_{\text {Node-B, } \max } \cdot(\mathrm{R} 4$ load $\left.) / 100\right)$ are depicted in Figure 2 . It can be seen that the average total cell data rates show a maximum around $3.5 \mathrm{~W}$ and the average HSDPA user data rate shows its maximum around $6.5 \mathrm{~W}$. Therefore, in case that the HSDSCH power is RNC controlled, the optimum power setting depending on the optimization criteria (either the average HSDPA data rate or the average cell data rate) can be found. Furthermore, it can be seen that in case of a ITU-R PedA channel profile, HSDPA offers a broad power operating range in which the throughput is nearly optimum.
TABLE I

SIMULATION PARAMETERS FOR FIGURE 2.

\begin{tabular}{llc}
\hline Parameter & & Value \\
\hline network & Node-B distance & $0.75 \mathrm{~km}$ \\
& max Node-B power & $43 \mathrm{dBm}$ \\
& $P_{\text {common }}$ & $33 \mathrm{dBm}$ \\
& shadow fading variance & $8 \mathrm{~dB}$ \\
& channel type & ITU-R PedA \\
HSDPA & assigned codes & 5 \\
& HS-DSCH power & variable \\
UE & category & 6 \\
& noise figure & $7 \mathrm{~dB}$ \\
& Rake fingers & 4 \\
Release 4 & load & fixed, $20 \%$ \\
& $\varrho$ & $5.2 \mathrm{~dB}$ \\
& $R$ & $384 \mathrm{kbit}$ \\
\hline
\end{tabular}

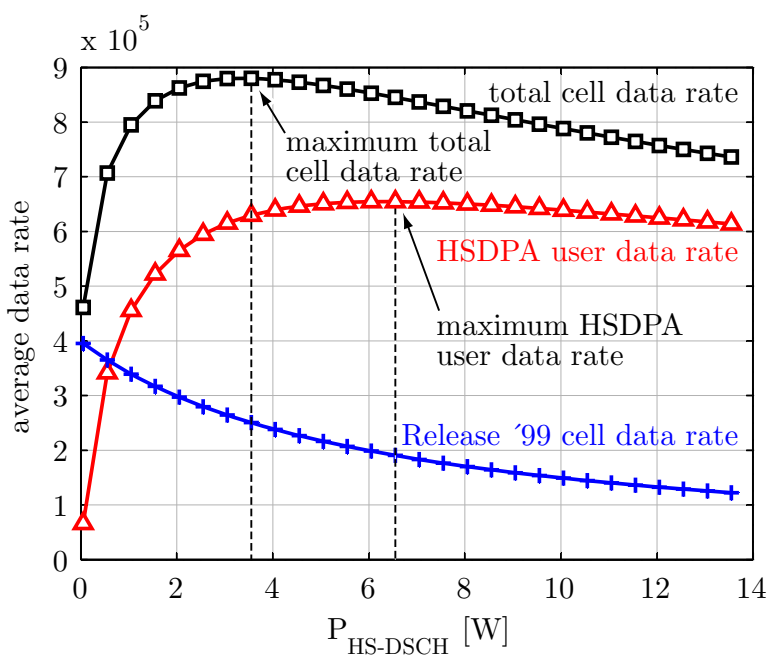

Fig. 2. Average data rates evaluated according to the simulation settings in Table I with RNC power control of the HS-DSCH.

By running a set of these simulations, we were able to analyse the relation between the HS-DSCH power level, at which the maximum of the average total cell data rate is achieved, and the Release 4 load of the cell. The results are illustrated in Figure 3, for different Node-B distances. It can be seen that the optimum HS-DSCH power scales with the Release 4 load, but then starts to drop down, which is caused by the limited maximum Node-B transmission power. It can be observed that the resulting curves do not differ a lot for the different cell sizes. This is because the transmission powers of the neighbouring cells are equally scaled with the transmission power of the serving Node-B and thus e.g. the shape of the total cell data rate is approximately only shifted up and down, but not further distorted. Accordingly, in the cell average, this results in nearly the same behavior.

\section{B. Node-B controlled HS-DSCH power}

After the investigation of the network performance in case of RNC controlled HS-DSCH power management, we also 


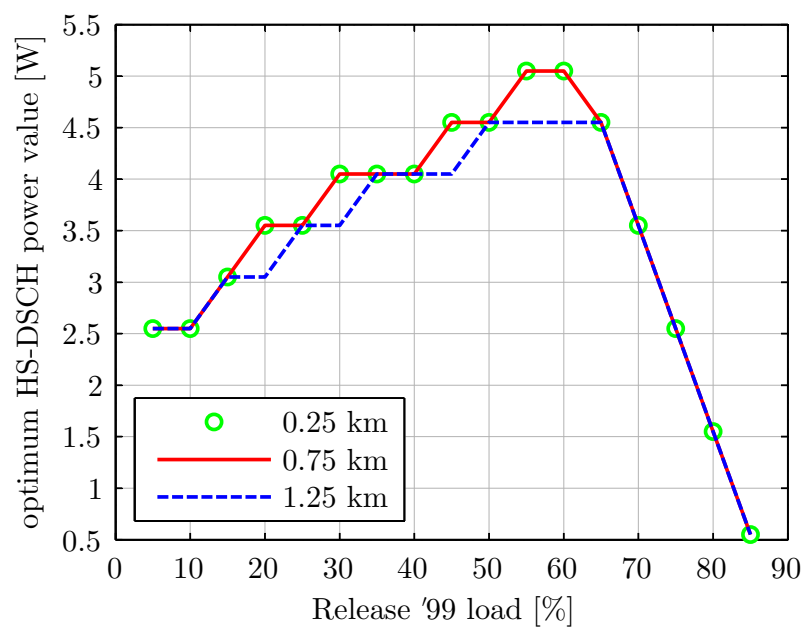

Fig. 3. Relation between the Release 4 load of the cell, and the according HSDSCH power that maximises the average total cell throughput. The simulation parameters are set to those in Table I, expect for the Node-B distance.

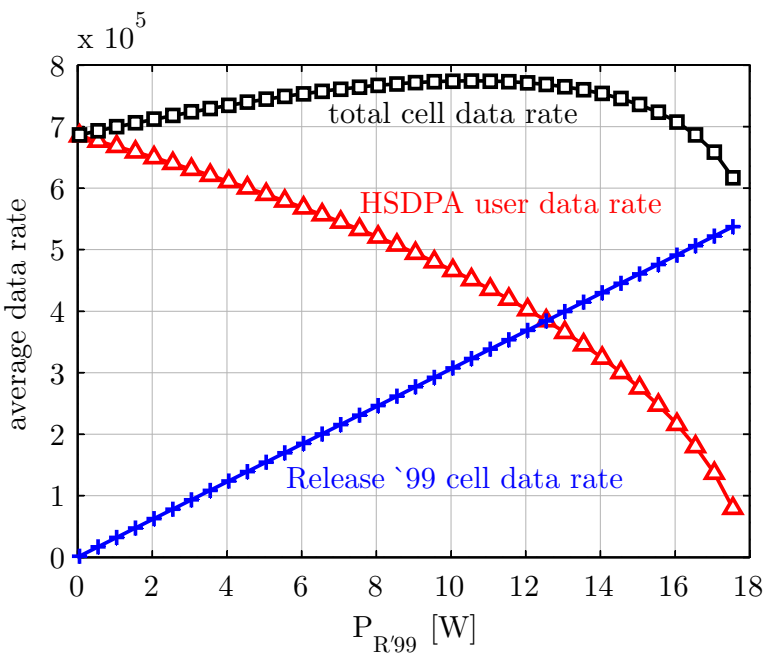

Fig. 4. Average data rates for fast Node-B power control of the HS-DSCH.

evaluated the behavior in case of fast Node-B HS-DSCH power control. The simulation parameters are nearly equal to the ones in Table I, only the Release 4 load is now variable.

The results of the simulations are shown in Figure 4. In contrast to the situation before, it can be seen that the optimum power distribution (in terms of the total cell data rate) is located at a higher amount of Release 4 traffic. Accordingly, it seems reasonable to "downgrade" users continuously observing a bad channel condition to a $384 \mathrm{kbit} / \mathrm{s}$ bearer, which is an optimizing criteria for the radio resource control (RRC) algorithms used within the network.

\section{CONCLUSIONS}

We have presented an HSDPA network performance evaluation in a network scenario where also Release 4 WCDMA traffic is present. The principal focus of the paper is on the achievable HSDPA data rates under such conditions, but by a coarse modeling of the Release 4 data rate we were able to investigate the overall network performance too. Our results show that optimum power settings exist, which lead to a maximum in the average cell data rate. Furthermore, we studied the differences of the network behavior in two different power control scenarios. The generated results offer a more sophisticated understanding of the network performance under the specified conditions and can serve for optimization of e.g. the RRC settings.

\section{ACKNOWLEDGEMENTS}

The authors would like to thank mobilkom Austria AG for supporting their research, in particular T. Ergoth and W. Wiedermann for numerous discussions and helpful suggestions. The views expressed in this paper are those of the authors and do not necessarily reflect the views within mobilkom Austria AG.

\section{REFERENCES}

[1] H. Holma and A. Toskala, WCDMA for UMTS - Radio Access For Third Generation Mobile Communications, 3rd ed. John Wiley \& Sons, Ltd, 2005.

[2] K. Pedersen, T. Lootsma, M. Stottrup, F. Frederiksen, T. Kolding, and P. Mogensen, "Network performance of mixed traffic on high speed downlink packet access and dedicated channels in WCDMA," in Proc. of the IEEE Vehicular Technology Conference 2004, Sep. 2004, pp. 44964500.

[3] Members of ITU, "Recommendation ITU-R M.1225: Guidelines for the evaluation of radio transmission technologies for IMT-2000," ITU, Tech. Rep., 1997.

[4] Members of COST 231, "Digital mobile radio towards future generation systems - COST 231 final report," COST, Tech. Rep., 1999.

[5] K. Brueninghaus, D. Astely, T. Salzer, S. Visuri, A. Alexiou, S. Karger, and G.-A. Seraji, "Link performance models for system level simulations of broadband radio access systems," in Proc. of the 16th International Symposium on Personal, Indoor and Mobile Radio Communications (PIMRIC) 2005, Sep. 2005, pp. 2306-2311.

[6] A. Seeger, M. Sikora, and A. Klein, "Variable orthogonality factor: a simple interface between link and system level simulation for high speed downlink packet access," in Proc. of the IEEE Vehicular Technology Conference 2003, Oct. 2003, pp. 2531-2534.

[7] Members of 3GPP, "Technical specification group radio access network; radio interface protocol architecture, 3GPP TS 25.301," 3GPP, Tech. Rep. v7.1.0, Mar. 2007.

[8] F. Brouwer, I. de Bruin, J. Silva, N. Souto, F. Cercas, and A. Correia, "Usage of link-level performance indicators for HSDPA network-level simulations in E-UMTS," in Proc. of the Eighth International Symposium on Spread Spectrum Techniques and Applications, Sep. 2004, pp. 844-848.

[9] Members of 3GPP, "Technical specification group radio access network; physical layer procedures (fdd), release 7, 3GPP TS 25.214," 3GPP, Tech. Rep. v7.4.0, Mar. 2007.

[10] R. Litjens, "HSDPA flow level performance and the impact of terminal mobility," in Proc. of the Wireless Communications and Networking Conference, Mar. 2005, pp. 1657-1663.

[11] TSG-RAN Working Group 4, "Revised HSDPA CQI proposal," 3GPP, Tech. Rep. R4-020612, Apr. 2002.

[12] K. Sipila, K.-C. Honkasalo, J. Laiho-Steffens, and A. Wacker, "Estimation of capacity and required transmission power of WCDMA downlink based on a downlink pole equation," in Proc. of the IEEE Vehicular Technology Conference 2000, May 2000, pp. 1002-1005.

[13] K. Pedersen and P. Michaelsen, "Algorithms and performance results for dynamic HSDPA resource allocation," in Proc. of the 64th Vehicular Technology Conference, Sep. 2006, pp. 1-5.

[14] Members of 3GPP, "Technical specification group radio access network; multiple input multiple output in utra," 3GPP, Tech. Rep. v7.0.0, Mar. 2007. 